$63^{\text {ème }}$ Congrès de la SFCO, 01004 (2015)

DOI:10.1051/sfco/20156301004

(C) Owned by the authors, published by EDP Sciences, 2015

\title{
CONFÉRENCE
}

\section{Facteurs Humains et erreurs médicales}

\section{Renouard F}

Paris, France

frenouard@aol.com

Comme pour toutes pratiques médicales, la pose d'implants dentaires conduit inévitablement à devoir faire face à des complications et à des échecs. Cependant, à ce jour, seuls les aspects techniques des complications sont évoqués. Ainsi en implantologie, de nouvelles formes d'implants, de nouvelles surfaces ou encore de nouveaux piliers sont proposés pour tenter d'améliorer le taux de succès des traitements implantaires. Mais on s'aperçoit qu'en pratique privée, le taux de complications ne diminuent par proportionnellement aux avancées technologiques.

Le comportement humain, très influencé par le stress, est rarement mis en cause dans la chaîne d'événements qui transforme une erreur en complication.

Dans les années 70, la prise en compte des facteurs humains a permis de réduire énormément le nombre d'accidents en aviation jusqu'à faire qu'en 2013, aucune compagnie aérienne européenne n'a eu à déplorer d'accidents. Le monde médical tarde à incorporer ces concepts dans son enseignement et dans sa pratique, bien que de très nombreuses publications en démontrent l'efficacité indiscutable. Il est ainsi montré que $80 \%$ des évènements indésirables graves en médecine sont liés à des erreurs humaines (Fabri et Zayas-Castro 2008). Le refus d'appliquer des protocoles établis, le refus de communiquer de façon efficace au sein de l'équipe, le refus d'utiliser les check listes, la présence de fort gradient d'autorité au sein des équipes, la surconfiance des cliniciens (Berner et Graber ML 2008) ou encore l'impossibilité de gérer son stress pendant les interventions (Wetzel et al. 2006) sont autant de facteurs qui vont avoir des impacts négatifs sur la sécurité des pratiques médicales. L'erreur médicale est considérée comme la première cause de décès à l'hôpital et la 5ème cause de décès toutes causes confondues. Le but de la présentation est de monter que le comportement des praticiens et de leurs équipes, que l'on appelle l'attitude, peut avoir des répercussions directes sur les taux de succès des procédures. La mise en œuvre de solutions simples, qui seront discutées pendant la présentation, permet de réduire de façon significative le nombre d'événements indésirables graves.

This is an Open Access article distributed under the terms of the Creative Commons Attribution License 4.0, which permits unrestricted use, distribution, and reproduction in any medium, provided the original work is properly cited. 